\title{
Itinerario terapéutico de las personas con discapacidad y mediaciones en el cuidado de la salud: la mirada de los familiares
}

| ${ }^{1}$ María Pía Venturiello |

Resumen: Las familias son agentes cualificados de las prácticas en salud y contribuyen en la búsqueda del cuidado de las personas con discapacidad. Este artículo presenta los itinerarios que recorren los adultos con discapacidad motriz en torno de su rehabilitación y la participación de sus familiares en este proceso. A tal fin se analizan las entrevistas semiestructuradas a familiares que viven con personas con discapacidad motriz de 50 años o más en el Área Metropolitana de Buenos Aires (AMBA). Para el relevamiento de la información de carácter cualitativo, se utilizó una muestra de tipo intencional (18 casos) por cuotas según sexo, nivel socioeconómico y tipo de vínculo (hijo/a o cónyuge). Los hallazgos muestran que los itinerarios terapéuticos en vistas del cuidado y la rehabilitación de las personas con discapacidad se dividen en dos etapas centrales: la búsqueda de un diagnóstico y la búsqueda del tratamiento. Ambas instancias se configuran de acuerdo a los factores sociales, económicos y culturales de sus familias. Según las evidencias se concluyó que los servicios de salud pueden funcionar, por momentos, como obstaculizadores de los itinerarios terapéuticos de sus usuarios si desatienden el vínculo entre sus acciones y la red familiar, en la cual se toman decisiones sobre el tratamiento a seguir y se gestiona cotidianamente el cuidado.

> Palabras-clave: itinerarios terapéuticos; discapacidad; cuidado; familia.
1 Instituto de Investigaciones Gino Germani. Facultad de Ciencias Sociales. Universidad de Buenos Aires. Doctoranda en Ciencias Sociales. Universidad de Buenos Aires. Correo electrónico: venturiello@yahoo. com.ar

Recibido: 17/10/2011. Aprobado: 13/08/2012. 


\section{Introducción}

Las familias son agentes cualificados de las prácticas en salud y contribuyen en la búsqueda del cuidado de las personas (BELLATO et al., 2009). En este sentido, los allegados de las personas con discapacidad intervienen facilitando y acompañando el proceso de búsqueda de atención en salud vinculada a la rehabilitación. Este artículo presenta los itinerarios terapéuticos que recorren los adultos con discapacidad motriz en torno de su rehabilitación, desde la perspectiva de los familiares que acompañan ese proceso.

La reflexión sobre la vinculación entre discapacidad y familia supone comprender al individuo en un tejido de interrelaciones. Tal abordaje resulta acorde con una perspectiva que considera los procesos de salud-enfermedadatención como resultado de condiciones históricas, económicas y culturales (GRIMBERG, 1998), donde ni la salud ni la enfermedad son entendidas como hechos objetivos e invariables, sino que están permeadas por las redes sociales y las instituciones. Del mismo modo, la discapacidad no se restringe a los aspectos fisiológicos sino que se define a partir de la falta de respuestas sociales ante ciertas condiciones físicas (OLIVER, 1998; FERREIRA, 2007), que incluyen la carencia de atención en salud.

Las personas delinean, junto a sus familias, los itinerarios terapéuticos, entendidos como las trayectorias de búsqueda, producción y gerenciamiento del cuidado de la salud (BELLATO et al., 2008). Los primeros estudios con esta perspectiva se centraron en el comportamiento del enfermo, analizando sus elecciones desde una óptica individualista y utilitarista. Una segunda vertiente, criticada por sus deficiencias teórico-metodológicas, focalizaba su argumentación en la influencia de los determinantes culturales sobre la utilización de los servicios de salud (TRAD et al., 2010).

Hacia la década de 1970, se comienzan a analizar los itinerarios terapéuticos contemplando los significados atribuidos a la enfermedad y el valor de las redes sociales (TRAD et al., 2010). Varios autores (CONRAD, 1982; KLEINMAN, 1980; FRIEDSON, 1978) plantearon que las significaciones acerca de la enfermedad y el rol del enfermo varían con la edad, el sexo, el rol familiar, la clase social, la educación y la ocupación. Estas connotaciones sobre el estar 
enfermo son construidas socialmente para dar sentido a padecimientos, hitos y acontecimientos en la vida de las personas y los grupos sociales. Es por esto que cuando a alguien se le diagnostica una enfermedad o se autodefine como enfermo, varía su comportamiento y el del resto de las personas de su entorno (CONRAD, 1982). Aunque la discapacidad puede o no estar relacionada a una enfermedad, la estigmatización social que se construye en torno de esta condición supone una redefinición de su identidad social y su comportamiento (FERREYRA, 2007).

En la búsqueda por el cuidado que realizan los sujetos dentro del "sistema de cuidado de la salud", Kleinman (1978) refiere a la existencia de tres subsistemas. El subsistema familiar, que incluye a las redes sociales y comunitarias más próximas, donde las enfermedades son primeramente detectadas y enfrentadas con un conocimiento no especializado. El subsistema profesional, donde la biomedicina es preponderante como la forma de cura reconocida legalmente. El subsistema popular, generalmente unido al subsistema familiar, consiste en especialistas que ofrecen curas no formales ni reconocidas legalmente.

Estos subsistemas sostienen interpretaciones divergentes sobre la enfermedad y la terapia. Al entrar en contacto a largo de los itinerarios terapéuticos se generan intercambios entre las diferentes concepciones (KLEINMAN, 1978). De acuerdo con Menéndez (2005), a partir de los recorridos de los sujetos en busca de atención en salud se pueden identificar todas las formas de atención que intervienen y cómo los usuarios las combinan de acuerdo a sus necesidades. En este sentido, los itinerarios terapéuticos suponen una secuencia de decisiones y negociaciones entre varias personas y grupos con interpretaciones diversas sobre la dolencia y la terapia adecuada a ésta (MATTOSINHO; SILVA, 2007). Asimismo, se orientan por la búsqueda de soluciones prácticas y de acuerdo a los quehaceres y las experiencias cotidianas que generan trayectorias singulares (ALVES; SOUZA, 1999).

En su llegada a los servicios de salud, los usuarios nunca se encuentran solos, sino que están acompañados por sus familiares o amigos. De allí la importancia de comprender a las personas en la trama de sus redes sociales que se constituyen a partir de los procesos de mutualidad en las cuales se recrean las solidaridades (MARTINS, 2008). En el seno de estas redes se construyen sentidos sobre la salud, la enfermedad y la discapacidad. Por ello es preciso comprender el 
comportamiento de las personas con discapacidad en el marco de los vínculos familiares de los que forman parte y donde se toman decisiones relativas a sus tratamientos. En efecto, cuando una persona se encuentra en una condición de salud crónica precisa el cuidado continuo y prolongado que brindan las familias, el cual se distingue de los cuidados puntuales que brindan los profesionales de la salud (BELLATO et al., 2009). Al respecto, Verdugo (2004) ha ampliado el concepto de calidad de vida de la persona con discapacidad hacia el de "familia con discapacidad". De acuerdo con esta definición, la familia constituye una pieza fundamental para el bienestar, la salud y la rehabilitación de esas personas y, al mismo tiempo, necesita contención y apoyos frente a escenarios que pueden resultar traumáticos y facilitar enfermedades.

De las reflexiones anteriores surgen los siguientes interrogantes: ¿cómo construyen sus itinerarios terapéuticos en torno a la rehabilitación las personas con discapacidad? ¿Cómo colaboran sus familiares en este proceso? ¿Qué aspectos subjetivos se ponen de manifiesto en estas búsquedas? ¿Qué obstáculos encuentran?

\section{Objetivos y metodología}

El objetivo de este artículo es describir las experiencias de los itinerarios terapéuticos de las personas con discapacidad desde la perspectiva de sus familiares. ${ }^{1}$ A tal fin se analizan las entrevistas semi-estructuradas a familiares que viven con personas con discapacidad motriz de 50 años o más en el Área Metropolitana de Buenos Aires (AMBA). El trabajo de campo se realizó entre los años 2010 y 2011. Para el relevamiento de la información, de carácter cualitativo, se utilizó una muestra de tipo intencional (18 casos) por cuotas según sexo, nivel socioeconómico y tipo de vínculo (hijo/a o cónyuge). Se analizaron dimensiones relativas a: tipo de vínculo; tipo de discapacidad del familiar; origen de esa discapacidad; necesidades de cuidados del familiar con discapacidad; apoyos y cuidados que brindan las familias, cómo acompañan, redes de apoyo informales y formales; vivencias en relación la rehabilitación de su familiar; acceso a los servicios de salud; relación con los profesionales de la salud.

La indagación se realizó bajo con el consentimiento expreso del entrevistado y conociendo el contenido de la guía de pautas. Se les informó acerca de los fines de la investigación. Se resguardó el anonimato de los entrevistados mediante la utilización de nombres de fantasía. 


\begin{tabular}{|l|l|}
\hline Residencia & $\begin{array}{l}6 \text { en la Ciudad Autónoma de Buenos Aires } \\
12 \text { en el Conurbano de Buenos Aires }\end{array}$ \\
\hline $\begin{array}{l}\text { Parentesco con la persona con } \\
\text { discapacidad }\end{array}$ & $\begin{array}{l}12 \text { cónyuges } \\
6 \text { hijos }\end{array}$ \\
\hline Máximo nivel educativo & 3 hasta primario completo \\
alcanzado & 2 secundario incompleto \\
7 secundario completo & 1 terciario incompleto \\
2 terciario completo \\
2 universitario incompleto \\
2 universitario completo \\
\hline Situación laboral & 4 no trabajan \\
& 2 desocupados \\
12 trabajos por cuenta propia como empleado, \\
operario o como empleada doméstica
\end{tabular}

* Obra social de jubilados y pensionados.

** Obra social para pensiones no contributivas o graciables de la Provincia de Buenos Aires.

\section{Rehabilitación e itinerarios terapéuticos}

Con el fin de tener una mayor comprensión de las trayectorias de las familias en los servicios de salud es preciso establecer el origen de la discapacidad de sus 
familiares. Según los testimonios de los entrevistados, aquéllos tuvieron diversas enfermedades con secuelas discapacitantes:

- Cuatro personas que tuvieron poliomielitis y una mujer con una afección congénita en la columna, viven con su discapacidad desde la infancia y han hecho la rehabilitación en esa etapa, aunque algunos la han vuelto a practicar durante la vida adulta, de acuerdo a sus necesidades de movilidad y los deterioros que conlleva el paso del tiempo. Una de las personas necesitó también intervenciones quirúrgicas.

- Tres personas sufrieron un accidente cerebro vascular hace cinco años y una hace quince. Las tres que sufrieron esa discapacidad recientemente aún se encuentran en la fase de rehabilitación y siguen percibiendo mejoras en su movilidad, en cambio la persona que padece esa enfermedad hace tiempo posee un cuadro complicado por una artrosis deformante.

- El resto de las personas padece enfermedades neurológicas o de otros tipos, para las cuales corresponden tratamientos con prescripciones acordes a las necesidades específicas. En estos casos, las enfermedades suelen generar secuelas que se manifiestan a medida que avanza el tiempo y que requieren rehabilitación para mejorar la calidad de vida.

\section{En búsqueda del diagnóstico}

Los itinerarios terapéuticos en vistas del cuidado y la rehabilitación de las personas con discapacidad se configuraron de acuerdo a los factores sociales, económicos y culturales de sus familias. En ese proceso se distinguieron dos etapas, en las cuales esos factores inciden: la primera, referida a la búsqueda de un diagnóstico, y la segunda, orientada al tratamiento.

Exceptuando los casos de poliomielitis y de luxación congénita, en las cuales el primer diagnóstico ocurrió en la infancia y dentro de la familia de origen, en el resto de los relatos los entrevistados han buscado junto a sus cónyuges y padres el diagnóstico de la enfermedad. Igualmente, aunque algunas personas se encuentren con discapacidad desde los primeros años de sus vidas, otras complicaciones agravaron esa condición a lo largo de su adultez, requiriendo de nuevos diagnósticos médicos.

Los itinerarios terapéuticos de estas personas están vinculados a las redes de las que forman parte los entrevistados. Ellos se caracterizan por prestar apoyos a lo 
largo de todo el proceso de búsqueda de atención (BELLATO et al., 2009). Así, la presencia de los entrevistados en los itinerarios terapéuticos de sus familiares es de una importancia impostergable. Ellos buscan, averiguan, hacen trámites, contienen y, sobre todo, se ocupan de la totalidad del proceso que atraviesa su ser querido. José expresa: "todo el arbitraje médico y asistencial lo tenemos que hacer nosotros".

La búsqueda de cuidados no supone acudir necesariamente a los servicios de salud. El primer paso hacia ello es la búsqueda de un diagnóstico en el subsistema profesional. En este punto se encuentran dos lógicas disímiles sobre la enfermedad y los modelos explicativos acerca de ésta y comienzan una serie de transacciones e intercambios entre el médico, el paciente y su familia (KLEINMAN et al., 1978).

El diagnóstico sobre el estado de la salud constituye un requerimiento para avanzar con un tratamiento. No obstante, una vez que se acude a los servicios de salud, encontrar un diagnóstico médico certero puede demorarse. En ocasiones, el desconocimiento de ciertas enfermedades y los diferentes enfoques de las especialidades de la medicina postergan el diagnóstico sobre la situación del paciente y los caminos a seguir para su recuperación. Sobre esto, Facundo, cuya madre padece una enfermedad neurológica, expresa: "los médicos no terminaban de establecer si era una discapacidad o no".

De tal suerte y coincidiendo con Bellato et al. (2009), a medida que las redes formales de salud son menos resolutivas, las personas afectadas y sus familias movilizan otros recursos y personas en el marco de sus redes informales vinculadas a ellas de las formas más variadas, para obtener alivio a sus malestares. María Elisa y su esposo hallaron el diagnóstico sobre el problema físico que lo importunaba mediante los consejos de unos amigos.

Así, relata:

Él está en el hospital [de rehabilitación] porque uno de los kinesiólogos es muy amigo nuestro, hemos hecho gimnasia con él hace mucho tiempo. Y cuando lo vio así, le dijo: "¿Por qué no viene a verme al hospital, Juan Carlos? A ver si podemos adelantar algo" [...] Lo estuvieron evaluando y le dice: "Yo le aconsejaría que vaya a ver a un neurólogo porque la forma que tiene de caminar usted... eso es más problema neurológico que problema de artrosis". Bueno, le hizo caso [...] y nos enteramos de que tenía este tumor en la médula.

De este modo, a medida que se presentan necesidades no resueltas en los servicios de salud se acude a las redes informales, que aportan al rumbo que toma el itinerario terapéutico. 
Los entrevistados participan junto al familiar que precisa de cuidados en las decisiones. Buscar un diagnóstico no implica un hallazgo que se resuelve de una vez para siempre, sino que supone comenzar un diálogo entre los profesionales de la salud y el paciente y sus familiares. Este encuentro entre los valores y formas de resolución del sistema popular y el sistema médico trazan el recorrido hacia un diagnóstico. Frente a las respuestas de los profesionales de la salud, las familias pueden aceptar o no sus indicaciones. Con el fin de avanzar hacia nuevas decisiones sobre el cuidado, las familias suelen buscar nuevas opiniones, estudiar y apropiarse del saber médico ante diagnósticos que no satisfacen sus inquietudes.

Frente a la falta de ofertas idóneas del sistema médico las familias toman mayor protagonismo en los itinerarios terapéuticos de sus familiares, tratando de establecer tanto diagnósticos como tratamientos. Las fallas de resolución del sistema médico pueden deberse a un vacío en el conocimiento biomédico sobre determinado malestar y/o a la falta de contemplación de las necesidades que la enfermedad y la discapacidad conlleva en la cotidianeidad y subjetividad del paciente y su familiar (KLEINMAN et al., 1978)

Uno de los casos paradigmáticos es el de Andrea, cuyo esposo padece las secuelas discapacitantes de la fibrodisplasia osificante (FOC), enfermedad considerada poco frecuente. La rareza de esta patología retrasó su diagnóstico y para obtenerlo debieron consultar a muchas especialistas. Andrea resume cómo conocieron la enfermedad de su marido:

Cuando yo lo conocí a él, no teníamos idea de la enfermedad. Más él, que no sabía nada de la enfermedad. Él fue una de las primeras personas que tuvo FOC acá. Mi suegra recorrió con él por todos los lugares, le aplicaban tratamientos que no eran acordes a la enfermedad de él porque lo complicaban más. Y después empezamos con esta campaña de buscar y buscar. Estando acá en la línea aérea conseguimos un médico que era de Estados Unidos y por tener la facilidad de trabajar acá hemos viajado. Se atendió con él durante veinte años y él mismo lo direccionó a la fundación que opera en Filadelfia para un mejor estudio. En Filadelfia conocimos al Dr. [...], él fue el que descubrió el gen que genera esto, en el 2003-2004.

El caso del marido de Andrea pone de manifiesto los recursos de los que hay que disponer para alcanzar un diagnóstico médico adecuado, búsqueda que se intensifica cuando no existen avances suficientes en el conocimiento de la enfermedad. En estas situaciones, la iniciativa familiar constituye el motor más potente para diagramar las redes que brinden alivios al malestar que afecta a 
la persona y su entorno. Contar con capital cultural, simbólico y económico (BOURDIEU, 1991) es clave para poder llevar adelante esta tarea que requiere consultar varias opiniones y consolidar la propia.

Parte de la tarea que realizan las familias en esta etapa de diagnóstico es instruirse sobre el malestar que aqueja la salud del integrante afectado. No es preciso que se trate de una enfermedad poco conocida para que los entrevistados tomen la decisión de comenzar a investigar el tema y elaborar una mirada más independiente respecto de los médicos. En este sentido, algunos entrevistados no delegan en ellos las decisiones más caras al cuidado de su familiar: frente a la incertidumbre, las dudas y las diversas opciones, prefieren estudiar por su cuenta de qué se trata tal o cual enfermedad y adecuar las decisiones a las miradas de la familia. Así, Tamara, cuyo marido tuvo un ACV, expresa:

Sí, me puse a investigar porque cuando te dicen -"sí, tiene un problema cerebeloso", -"si, tiene esto", -“no, no se recupera más", porque hemos ido a tantos lugares que todos tienen distintas versiones, y digo, -"no cómo, ¿̨cómo no se va a poder recuperar?" Nosotros tenemos fe de que sí se va a recuperar, soñamos toda la familia de que va a caminar, tenemos esa esperanza, entonces me digo, -"no, tengo que averiguar".

Por su parte, Juan Oscar, cuya esposa padece una enfermedad neurológica explica:

Nunca se sabe bien qué es lo que pasa porque todos los días escucho algo nuevo, me meto en internet y encuentro algo nuevo, ¿viste algo que cabe en todas las cajas? Esto es así. Mirás y hace 20 años atrás nos decían que era una enfermedad desmielinizante, yo nunca había escuchado hablar de la mielina. Me interesé, estudié un poco el tema, eso te da una justificiación lógica. Nos decían que tenía cura, que no tenía cura...

Por su parte, Tamara, cuyo esposo sufre las secuelas de un ACV, cuenta sobre la rehabilitación de su marido y jerarquiza la gestión de la familia por sobre la ayuda de los médicos: "No, no, ellos (médicos) no nos dijeron nada (sobre el tratamiento de equinoterapia), uno tiene que averiguar si es lo que quiere hacer, eso me he dado cuenta y es lo que hice, averigüé, busqué y conseguí".

Tales relatos dan cuenta de la manera en que llegan a involucrarse los entrevistados para colaborar con la recuperación de sus familiares. La búsqueda de conocimiento también se asocia a una búsqueda por el sentido de lo que les sucede, siendo "la justificación lógica" y la "fe en la recuperación" alicientes en los momentos de mayor desazón. Poder comprender lo que ocurre dentro de una trama mayor de sentido implica una necesidad esencial al ser humano. Su 
1072 búsqueda se canaliza en el subsistema popular donde se atienden los aspectos subjetivos y la forma en que se experimenta la enfermedad y la discapacidad. En efecto, en las trayectorias del cuidado la biomedicina no es el único saber al que se recurre. Las creencias orientan búsquedas que incorporan el pensamiento mágico y, en algunos casos, se asiste a "curanderos" para aliviar los malestares. La situación de discapacidad y enfermedad implica cuestionamientos a las subjetividades individuales y colectivas, donde han de replantearse tanto los proyectos personales como familiares. Cuidar la salud de alguien es más que construir un objeto para intervenir sobre él y requiere considerar y construir proyectos que permitan a las personas alcanzar un equilibrio entre cuerpo, alma y espíritu (AYRES, 2001, p. 71 - citado en LACERDA; VALLA, 2008). En tal sentido, la vigencia de las concepciones que asocian los malestares del cuerpo a los daños del alma se ponen en juego en estas consultas. Así, Ana comenta:

[...] acompañarlo a algún médico especialista o a algún curandero, porque también tuvimos la época de un curandero... yo nunca estuve de acuerdo, pero si él quería ir, íbamos (...) pero uno que viene y te da un yuyito y te cura no, yo no te lo creo. Entiendo que por ahí él se aferra a cualquier cosa. Son épocas. Ahora con eso estamos bastante bien.

Juan Oscar relata una experiencia similar

La he llevado a curanderos, la he llevado a los que trabajan con flores de Bach, he ido a los lugares más insólitos, pobre María. Una vez la llevé a Laferrere, que vinieron los indios de Los Toldos, esos que son famosos hueseros. Otra vez fuimos a ver uno de esos que hacían las operaciones Filipinas, sin sangre, un verso de aquellos [...] y ya te digo, a mí me decían que algo servía y la llevaba.

Estas experiencias son paralelas a las búsquedas de diagnóstico en los servicios de salud. Buscar el cuidado supone atender a diferentes aspectos que se ponen un juego simultáneamente ante una discapacidad, pero se les otorga valores desiguales en cada subsistema: el familiar y el popular son los más sensibles a los aspectos subjetivos.

\section{En la búsqueda del tratamiento}

La búsqueda del tratamiento que las familias emprenden está estrechamente ligada a sus posibilidades económicas. Contar con cobertura prepaga y con la posibilidad de pagar los consultorios y servicios privados es determinante en el acceso a ciertos tratamientos. Sin estos recursos, las opciones para la rehabilitación 
se estrechan y los tiempos de espera para recibir cuidados idóneos se hacen más extensos. De este modo, Jessica expresa sobre su esposo con secuelas de ACV: "ya tendría que estar rehabilitado pero no se pudo por carencias económicas". Juan Oscar refiere sobre su esposa, que padece una enfermedad neurológica: "me gustaría llevarla a natación, pero no puedo pagar”.

Los gastos que requiere asistir a una persona con discapacidad son muy altos puesto que deben incluir, entre otras cosas, los traslados, prótesis e insumos, como por ejemplo pañales, lo cual implica contar con recursos de los que varios entrevistados no disponen. Frente a ello, la opción por la atención pública y la entrega gratuita de suministros no siempre colma las necesidades de quien las solicita. A veces contar con una cobertura privada tampoco soluciona estos menesteres. Más bien es el capital económico de cada familia lo que permite resolver la contratación de un servicio o la compra de un insumo, cuando los utilizados habitualmente resultan deficientes.

\section{¿Cómo son los tratamiento que realizan y cómo llegaron a ellos?}

Los familiares de los entrevistados hacen rehabilitación en centros públicos, privados o realizan sesiones de kinesiología en el hogar o en el consultorio de los profesionales. En algunos casos complementan la rehabilitación en varios lugares y diversas instituciones. A lo largo de sus itinerarios terapéuticos la rehabilitación abarca actividades variadas: ejercicios con aparatos, kinesiología, terapia ocupacional, natación, pintura y equinoterapia. Esta multiplicidad de tareas está dirigida a lograr una mayor autonomía de la persona con discapacidad.

Elaborar un esquema de actividades para mejorar el bienestar de la persona con discapacidad y su familia lleva tiempo de búsqueda y maduración. Aún así, las personas de niveles socioeconómicos bajos no logran cubrir todas sus necesidades de atención. Así, nuevamente Jessica expresa: "Si fuera por mí, a la kinesióloga lo quiero llevar cuatro veces, pero tengo que llevarlo dos veces porque pago el remis y a la kinesióloga”. El lugar de residencia también condiciona el acceso a los servicios de salud, a los centros de rehabilitación y a los hospitales más cercanos. Los entrevistados de zonas desfavorecidas encuentran en sus jurisdicciones menos ofertas para la rehabilitación. 
Asimismo, debe tenerse en cuenta que el tratamiento de rehabilitación puede variar según las etapas de la enfermedad y la discapacidad, así como diferir en sus resultados según el tipo de afección física. Por ejemplo, entre las personas que han sufrido un ACV pueden alcanzarse avances significativos en la movilidad del cuerpo, mientras que en las personas que padecen determinadas enfermedades neurológicas el propósito de la rehabilitación es evitar un avance agresivo. Ambas situaciones impactan en las expectativas sobre el tratamiento de manera desigual. En tal sentido, Olivia expresa su opinión sobre la rehabilitación que realiza su pareja tras un ACV: "Sí, ¡avanzó muchísimo! pero tiene mucha fuerza de voluntad. No se dejó estar”.

Analía tiene otra perspectiva sobre la situación de su marido: "El médico lo atendió a Juan en las primeras etapas, fuimos, lo acompañé. Lo atendió y le dijo: esto va a ser así, no busque otra cosa, la esclerosis múltiple es así, conviene que haga rehabilitación. ¿Cómo termina? En silla de ruedas”. La forma en que se vivencian los malestares pueden ser muy diversas y requieren cuidados acordes a ellas. La pluralidad de estas interpretaciones también varía de acuerdo a los recursos con los que cuentan las familias y la relación que entablan con los especialistas (MATTOSINHO; SILVA, 2007).

Al momento de buscar un tratamiento o una institución donde hacer rehabilitación se valoran aquellos que ofrecen espacios de recreación con vistas a mejorar la calidad de vida de la persona con discapacidad y su familia. En general, en los servicios de salud, las dimensiones relacionadas a los contextos de vida de los usuarios no se contemplan (MANGIA; MURAMOTO, 2008). De allí que cuando el vínculo entre la persona con discapacidad y su entorno es tenido en cuenta por la institución a la que se acude, esto es muy valorado.

Juan expresa sobre el instituto público donde hace rehabilitación su esposa, quien vive con las secuelas de la poliomielitis:

Acá el instituto tiene la política de que cada uno tiene que valerse por sí mismo. Eso es lo que yo tenía pensado también, eso me ayuda mucho. Aprender a nadar la hizo más segura, ella no sabía nadar, le tenía miedo al agua, ahora se mete hasta los cuatro metros. [...] Ella se hizo muy independiente acá, más la pintura, todo eso que está haciendo.

De este modo, además de satisfacer las necesidades de ejercicios físicos, acudir a instituciones con una mirada más integral puede otorgar una serie de 
beneficios adicionales al fortalecimiento del cuerpo. Las ayudas para resolver la vida cotidiana y los problemas prácticos con los que deben enfrentarse las personas con discapacidad y su familia son destacadas por los entrevistados. Estas atenciones en salud ofrecen una asistencia integral que dan respuestas a las necesidades de cuidados de los usuarios.

Ana explica: "Ahora fuimos a este instituto [...] Fue todo un tratamiento super intensivo. Nos dijeron de entrada: no se va a curar pero le va a servir para muchas cosas. Te enseñan a manejarte con la silla de ruedas, cuál es la mejor silla de ruedas, cómo manejar y tener más independencia”.

María Elvira considera la asistencia de su marido al hospital de rehabilitación de su barrio como una manera de esparcimiento: "Le hace bien, no solo físicamente, sino el ambiente que se vive ahí adentro. Él va allí, joroba, van con otro señor y dicen que van a hacer un equipo de fútbol, el equipo de los rengos, o ese tipo de cosas".

\section{Relación con el médico}

La importancia de la escucha por parte de los médicos se debe a dos aspectos: en primer lugar supone una manera de cuidado hacia la subjetividad de la persona que padece cierto malestar (AYRES, 2001, p. 71 - citado en LACERDA; VALLA, 2008). Para los entrevistados, los médicos son referentes fundamentales en el cuidado de la salud del familiar con discapacidad. De ellos no solo esperan prescripciones para el tratamiento sino también una atención cálida y comprensiva de su situación. En tal sentido, los entrevistados suelen estar conformes con los médicos que atienden a sus familiares cuando su trato es "humano", "solidario" y se muestran afectuosos.

En segundo lugar, incorporar las narrativas en la atención a los usuarios de los servicios de salud permite conocer sus experiencias singulares y, a partir de ellas, establecer estrategias de cuidados vinculadas a las situaciones en las que se encuentran y las necesidades que deben atender (MANGIA; MURAMOTO, 2008). En este sentido, cuando se contempla la red familiar dentro de la cual se proveerán los cuidados, los entrevistados se muestran conformes. Una de las prácticas que valoran es la transmisión de pautas prácticas para la mejor atención de sus familiares. Así, Olivia comenta la estadía en el hospital donde estuvo internada su pareja por un ACV: 
¡la calidad humana que hay en ese hospital es increíble! Hacen todo humanamente, con amor, con pocos elementos. Decir "dejá", "mirá", "te voy a enseñar", "esto se hace así", para cuando tengas que bañarlo en tu casa y un enfermero viene y hacerlo conmigo para enseñarme a higienizarlo. Este, ir a pedir algo difícil y decir, "no te preocupes mi amor", esto es normal "hacé así”, "hacé asá”.

A la inversa, algunas experiencias que los entrevistados relatan refieren a cuando sus familiares son atendidos sin contemplar sus sentimientos, emociones y cómo se ve afectada su subjetividad ante la situación de salud que deben encarar. Alicia expresa que su esposo con discapacidad no quiere ir a los médicos porque "está cansado de ser un conejo de clínicas”. Otros tienen una mirada más ambigua. Ana afirma que el trato con los médicos está: "a veces mal, a veces bien”.

La relación de asimetría que se establece entre el médico y sus pacientes (MENÉNDEZ, 2005) a partir del saber especializado del primero y la vulnerabilidad de los segundos, puede adquirir diversos matices. Los entrevistados más informados sobre el estado de salud de su familiar tienden a establecer un trato con una dinámica diferente a raíz de la adquisición de saberes médicos. Así Andrea, cuyo esposo sufre una enfermedad muy poco conocida, comenta:

yo me siento con los médicos. Por ejemplo, él desde hace cinco años tiene un cálculo en la vejiga muy importante, que cualquiera que lo ve dice no sé cómo esto está ahí adentro. Y yo me he sentado con el urólogo, que es uno de los mejores que hay en Buenos Aires y así, analizando qué se podía hacer y qué no. El bajando libros de su biblioteca. Yo porque me metí de lleno, y puedo hablar con los médicos al mismo nivel. Y me escuchan porque no conocen sobre la enfermedad ¿Qué le va a hacer? Eso no. Pongo el punto yo, porque si no le podés generar desastre. Pero tenés que saber mucho. Tenés que estar al tanto.

\section{Obstáculos en las redes de la salud: escasez de información, resistencias burocráticas y fallas en los servicios}

Los itinerarios terapéuticos suponen un recorrido donde las redes sociales proveen diversos apoyos, entre ellos, generar información precisa para acceder a los servicios de salud. Esta información consiste en datos sobre dónde atenderse, sobre la gratuidad del servicio, cuáles son los tratamientos disponibles, cuáles están cubiertos por la seguridad social, qué derechos amparan el acceso a esos servicios, entre otros. Tales datos pueden constituirse en facilitadores $\mathrm{u}$ obstaculizadores de la consolidación de las redes sociales y especialmente de aquellas que son institucionales o formales (MARTINS, 2008). 
La información sobre los lugares en que las personas pueden atenderse puede constituirse en un bien que hay que saber encontrar, puesto que no siempre se presenta de manera espontánea. De tal suerte, Tamara expresa cómo llegó al centro donde su marido hace rehabilitación tras un año de búsqueda y luego de pasar por una obra social, una prepaga y perder la atención con el neurólogo que lo estaba tratando: "fue en una clínica para tratamiento de traumatología. Y entonces me dice [la empleada], -"lo que él necesita, acá no se hace, pero tengo un número de teléfono y averíguate, porque es de un centro de discapacidad”.

Una vez que su marido comenzó a atenderse comenta sobre los profesionales del centro: "Medio me llamaron la atención porque había pasado un año y medio del accidente, lo que pasa es que yo no conocía. Una vez que yo conocí empecé acá a venir, y venir, y venir, y ahora conozco a los médicos, me movilizo más".

El caso opuesto es el de Olivia, cuya pareja estuvo internada en el mismo hospital público y contó con el asesoramiento de unas trabajadoras sociales, quienes les indicaron dónde ir. Esta situación marcó una diferencia en el itinerario terapéutico de su familiar facilitando la llegada del cuidado. Tal contingencia evidencia que la información es un requisito para que las personas puedan desenvolverse adecuadamente dentro de los servicios de salud y accedan al cuidado que necesitan.

Los incumplimientos de las prestaciones que deben brindar las prepagas, obras sociales y hospitales públicos también constituyen obstaculizadores en el acceso al cuidado. Entre las decisiones que los entrevistados deben tomar junto a su familiar con discapacidad acerca del tratamiento a seguir, se encuentran las estrategias ante la evasiva de las prestadoras a cumplir con sus obligaciones.

Aunque la legislación sobre discapacidad se expide sobre las prestaciones que las prepagas y las obras sociales han de ofrecer a las personas que presentan el certificado de discapacidad, todos los entrevistados debieron insistir, muchas veces hasta el hartazgo, para que sus familiares las percibieran. De tal suerte, obtener sesiones ilimitadas de kinesiología, lo que debiera ser un trámite menor, puede implicar situaciones de tensión y presión por parte de los usuarios, a fin de que se cumplan sus derechos. Asimismo, algunos entrevistados se refirieron a la brevedad del tiempo de la sesión del kinesiólogo que atiende a domicilio. Así, Ana relata: Sabemos que a los kinesiólogos de las prepagas les pagan muy mal [...] vienen y te dan veinte minutos, y es como nada [...] no es una cosa muy intensiva como por ahí a él le gustaría”. 
Jessica comenta sobre un centro público "Porque allá no es que lo atienden al paciente. Allá entran 20 pacientes y lo tienen haciendo la fila, entonces van por quince minutos y ya está, terminó”.

Otro obstáculo, que se constituye en una cruzada a librar implica poder obtener sillas de ruedas motorizadas o adecuadas a las características físicas y de salud de cada usuario. Sobre ello Ana refiere el caso de su esposo: "Ya le dijeron que esa silla la tiene que cambiar. Por eso ahora estamos pensando en una dual que estamos tratando de que la prepaga la pague, ya dijeron que no, así que ahora lo vamos a consultar con una abogada".

Marcelo también se encuentra en un litigio legal con la obra social de su madre por un problema en la entrega de la silla de ruedas. Esta política de resistencia por parte de prepagas y obras sociales a entregar las sillas de ruedas adecuadas a la solicitud del usuario, demorándola y ofreciendo una más sencilla y menos onerosa, evidencia que subyace un conflicto de intereses económicos.

En una línea similar, Griselda explica las vicisitudes de la obra social de jubilados de su padre: "La primer silla la conseguimos por intermedio de unas personas allegadas y la otra la consiguió mi hermano. PAMI no, olvidate. Y para los pañales recién ahora nos dieron. Pero son unos pañales que no vale ni ponérselos [...] es un pañal malísimo". Asimismo, cuenta que estuvieron durante dos años esperando la pierna ortopédica para su padre.

Los retrasos en la entrega, así como el envío de productos de mala calidad, son prácticas que tienden a empeorar la calidad de vida del usuario y su familia quienes deben buscar soluciones a ello. Por ejemplo José relata: "pedimos la silla de ruedas a la prepaga, trámite va, trámite viene, al final Martín se pudrió y compró una silla. Cuando él la compra viene la de la prepaga”

Las personas que acuden a los servicios públicos tienen menos posibilidades de reacción. Allí, la falta de elementos y suministros -aparatos para hacer ejercicios, espacio para el salón de rehabilitación- es uno de los aspectos que los entrevistados señalan como perjudiciales para la buena atención. La excesiva burocracia para obtener descuentos en los medicamentos es otro de los aspectos señalados. Un problema mayor es la impuntualidad de las combis que llevan y traen a las personas desde el hogar a los centros de rehabilitación.

Así, Olivia relata sobre el centro al que acompaña a su pareja dos días por semana Acá es un poco a los ponchazos las cosas, por ejemplo, acá son cuatro las combis que hay para llevarlos y traerlos para rehabilitación. Y no alcanza, van a horarios diferentes, 
por ejemplo te dicen -"vamos a estar a las 10 de la mañana"-, entonces el discapacitado

dice -"no vamos a almorzar porque vienen a las 10"-, y se hace la una y no están, entonces el discapacitado está sin almorzar, está débil todo el día para hacer la gimnasia.

Estos testimonios evidencian fallas de infraestructura que impiden contemplar cabalmente la situación de la personas con discapacidad y la de su familia. En estos casos se genera malestar en el paciente que espera recibir cuidados, pérdida de tiempo y desorganización del esquema familiar.

Asimismo, la dinámica de puja que adquiere la entrega de sillas de ruedas, prótesis y medicamentos en forma gratuita no se corresponde con las obligaciones que deberían cumplir las prepagas y obras sociales. José lo resume de este modo al comparar las obras sociales nacionales con las de Francia, donde vivió: "los servicios de obra social tienen un toque de beneficentes antes que de deber [en Argentina], esa es la diferencia que yo veía con los franceses, ahí nadie te hace un favor".

\section{Comentarios finales}

En este trabajo se analizó el modo en que se configuran los itinerarios terapéuticos de las personas con discapacidad desde la perspectiva de sus familiares, esperando hacer un aporte para la mejora del cuidado en los servicios de salud. En este sentido, se evidenciaron la necesidad de tratamientos de rehabilitación con un enfoque integral del usuario e inmerso en una red familiar. Contemplar las diferentes dimensiones de la subjetividad afectadas ante la discapacidad resulta igualmente importante y, en muchos casos, supone acudir en busca de alivios al subsistema de salud popular y las redes informales. Asimismo, se evidenció que el sector de servicios de salud impone obstáculos al cuidado de las personas con discapacidad a causa de sus incumplimientos.

Analizar las formas de atención desde la perspectiva de los sujetos permite conocer, en mayor medida que si se parte desde los curadores, cuáles son los diferentes cuidados que se demandan y de qué modo van delineando la trayectoria hacia su obtención (MENÉNDEZ, 2005).

Los itinerarios terapéuticos que transitan las personas con discapacidad junto a sus familiares implican una sucesión de decisiones y negociaciones. Estas se vinculan a la búsqueda de un diagnóstico y un tratamiento que les brinden los cuidados adecuados. Satisfacer estas necesidades conlleva recursos para poder solventar económicamente los tratamientos. Asimismo, requiere contar con las 
herramientas educativas que permitan combinar los distintos conocimientos que caracterizan a cada subsistema de salud (familiar, popular y profesional) (KLEINMAN, 1978). En la búsqueda de atención las personas con discapacidad y sus allegados parten del subsistema familiar, principal red de apoyos para el cuidado que está presente a lo largo del todo el itinerario terapéutico (BELLATO et al., 2009), y junto con el cual se accederá a los otros subsistemas. El subsistema popular asiste en la búsqueda de sentido que aqueja a los entrevistados y sus familiares, puesto que una discapacidad suele implicar una crisis en la identidad personal. Se consulta a estas redes en la búsqueda de mejoras o alivios en forma paralela a las consultas biomédicas. Por último, acudir al subsistema profesional implica comenzar una serie de transacciones (KLEINMAN, 1978), entre los conocimientos cotidianos que tienen las familias sobre la necesidad de la personas con discapacidad y los saberes sobre la afección con los que cuentan los médicos. A fin de consolidar criterios sobre qué tratamiento conviene seguir a su familiar con discapacidad, los entrevistados se dedican a investigar sobre la dolencia que aqueja a su ser querido. Esta aproximación al saber biomédico les otorga mayor autonomía sobre las recomendaciones de los profesionales y, de esta forma, equilibran los diferentes sentidos sobre la enfermedad y la discapacidad que sostienen en uno y otro subsector, a fin de mejorar algunos aspectos de su vida cotidiana.

Cuando las transacciones se dan en el otro sentido (desde el subsistema familiar al profesional) y los usuarios son escuchados por los profesionales, sus familiares expresan sentirse más cuidados en términos afectivos y en la resolución de sus necesidades diarias. De esta forma, valoran los tratamientos que trabajan sobre la autonomía del usuario, puesto que facilitan su desenvolvimiento y el de su familia, que debe asistirlo en menos actividades. Las redes del cuidado, que atraviesan los tres subsistemas, encuentran ciertos obstáculos en la circulación de los apoyos. Estos inhibidores de las redes del cuidado (MARTINS, 2009), se refieren a la falta de información necesaria para acceder a los servicios, las demoras burocráticas y las resistencias a brindar prestaciones que están garantizadas por ley.

La descripción realizada sobre los itinerarios terapéuticos constituye información acerca de cuáles son las necesidades que los usuarios deben satisfacer, dónde las buscan, las dificultades para alcanzarlas y las repuestas que ante ello brindan los servicios de salud. En el caso analizado se trata de acciones parciales 
que no contemplan la totalidad del problema que enfrentan las personas con discapacidad y sus familias. Teniendo en cuenta estos hallazgos y coincidiendo con Alves y Souza (1999), el conocimiento y la utilización de la información sobre los itinerarios terapéuticos por parte de las profesionales y los servicios de salud constituye una oportunidad para ofrecer cuidados más acordes a las necesidades de los usuarios y sus familias.

\section{Referencias}

ALVES, P.C.; SOUZA, I.M. Escolha e avaliação de tratamento para problemas de saúde: consideraçōes sobre itinerario terapêutico. In: RABELO, M.C.M.; ALVES, P.C.B.; SOUZA, I.M.A. (Org.). Experiência de doença e narrativa. Rio de Janeiro: Fiocruz, 1999. p.125-138.

BELlATO, R.; ARAUJO, L.F.S.; CASTRO, P. O itinerário terapêutico como uma tecnologia avaliativa da integralidade em saúde. In: PINHEIRO, R.; SILVA JUNIOR, A.G.; MATTOS, R.A. (Org.) Atenção básica e integralidade: contribuiçōes para estudos de práticas avaliativas em saúde. Rio de Janeiro: CEPESC, 2008. p. 167-187.

BELLATO, R. et al. Itinerários terapêuticos de famílias e redes para o cuidado na condição crônica: alguns pressupostos. In: PINHEIRO, R.; MARTINS, P.H. (Org.) Avaliaçãa em saúde na perspectiva do usuário: abordagem multicêntrica. Rio de Janeiro: CEPESC, 2009, p. $187-194$.

BOURDIEU, P. El sentido práctico. Madrid: Taurus, 1991.

CONRAD, P. Sobre la medicalización de la anormalidad y el control social. In: INGLEBY, D. (Ed.) Psiquiatría Crítica. La politica de la salud mental. Barcelona, Grijalbo, 1982. p. 129-154.

FERREIRA, M. Prácticas sociales, identidad y estratificación: tres vértices de un hecho social, la discapacidad. Revista Intersticios, v. 1, n. 2, 2007. Disponible en: http://www. intersticios.es/article/view/1084/854.

FREIDSON, E. La profesión médica. Península: Barcelona, 1978.

GRIMBERG, M. VIH/SIDA y proceso salud-enfermedad-atención: Construcción social y relaciones de hegemonía. In: Seminario Taller de Capacitación de Formadores. Buenos Aires: LUSIDA, 1998.

KLEINMAN, A. Patients and healers in the context of culture. An exploration of the borderland between anthropology, medicine and psychiatry. California: University of California Press, 1980.

Kleinman, A.; EISENBERG, L.; GOOD, B. Culture, Illness, and Care. Clinical Lessons from Anthropologic and Cross-Cultural Research. Annals of Internal Medicine, v. 88, n. 2, febrero, p. 251-258, 1978. 
LACERDA, A.; VALLA, V.V. As práticas terapêuticas de cuidado integral a saúde como proposta para aliviar o sofrimento. In: PINHEIRO, R; MATTOS, R.A. (Org.) Cuidado: as fronteiras da Integralidade. Rio de Janeiro: CEPESC, 2004.

MÂNGIA, E.F.; MURAMOTO, M.T. Itinerários terapêuticos e construção de projetos terapêuticos cuidadores. Revista Terapia Ocupacional. Universidad do São Paulo, v. 19, n. 3, set/dez, p. 176-182, 2008.

MARTINS, P.H. Usuarios, redes de mediadores e associaçoes públicas híbridas na saúde. In: PINHEIRO, R.; MATTOS, R.A. (Org.) Cuidar do cuidado: responsabilidade com a integralidade das açoes de saúde. Rio de Janerio: Cepesc, 2008.

MATTOSINHO, M.M.S.; SILVA, D.M.G.V. El itinerario terapéutico del adolescente con diabetes mellitus tipo I y sus familiares. Rev Latino-am Enfermagem, v. 15, n. 6, nov-dic, p. 1113-1119, 2007.

MENÉNDEZ, E. Intencionalidad experiencia y función: la articulación de los saberes médicos. Revista de Antropología Social, n. 14, p. 33-69, 2005.

OLIVER, M. ¿Una sociología de la discapacidad o una sociología discapacitada? In: BARTON, L (Comp.). Discapacidady Sociedad. Madrid: Morata-Fundación Paideia, 1998, p. 34-58.

TRAD, L.A.B. et al. Itinerários terapêuticos face à hipertensão arterial em famílias de classe popular. Cadernos Saúde Pública, Rio de Janeiro, v. 26, n. 4, Abril, p. 797-806, 2010.

VERDUGO ALONSO, M. Calidad de vida y calidad de vida familiar. In: Congreso Internacional de Discapacidad Intelectual, II. Enfoques y Realidad: Un Desafío. Medellín, Colombia; 23 al 25 sept 2004.

\section{Nota}

${ }^{1}$ Este artículo forma parte de la investigación para la tesis doctoral en desarrollo Los adultos con discapacidad motriz: percepciones y estrategias frente a los obstáculos sociales en el marco de sus redes familiares en el Área Metropolitana de Buenos Aires, con beca de finalización de doctorado del Consejo Nacional de Investigaciones Científicas y Técnicas (CONICET). Ministerio de Ciencia, Tecnología e Innovación Productiva. Presidencia de la Nación Argentina. 


\section{Abstract}

Itinerary therapeutic for people with disabilities and mediation in health care: the viewpoint of relatives

Families are qualified agents of health practices and contribute in the search for care of people with disabilities. This paper presents the itineraries taken by adults with mobility disabilities around their rehabilitation and the participation of their families in this process. To this end we analyzed semi-structured interviews with family members living with physically disabled people aged 50 or more in the Buenos Aires Metropolitan Area. For the survey of the qualitative information, we used a sample of intentional type (18 cases) according to sex, socioeconomic status and link (child / spouse). The findings show that the therapeutic in view of the care and rehabilitation of people with disabilities are divided into two main stages: the search for a diagnosis and seeking treatment. Both instances are configured according to the social, economic and cultural rights of the families. According to the evidence we concluded that health services can work, at times, as obstacles to the therapeutic itinerary of users and disregard the link between their actions and the family network, in which decisions about treatment options are taken and daily care is managed.

> Key words: therapeutic itineraries; disability; health care; family. 